\title{
HOMOGENIZATION OF MICROMAGNETICS LARGE BODIES
}

\author{
Giovanni Pisante ${ }^{1,2}$
}

\begin{abstract}
A homogenization problem related to the micromagnetic energy functional is studied. In particular, the existence of the integral representation for the homogenized limit of a family of energies

$$
\mathcal{E}_{\varepsilon}(m)=\int_{\Omega} \phi\left(x, \frac{x}{\varepsilon}, m(x)\right) \mathrm{d} x-\int_{\Omega} h_{e}(x) \cdot m(x) \mathrm{d} x+\frac{1}{2} \int_{\mathbb{R}^{3}}|\nabla u(x)|^{2} \mathrm{~d} x
$$
\end{abstract}

of a large ferromagnetic body is obtained.

Mathematics Subject Classification. 35B27, 74Q99, 82D40.

Received June 19, 2003.

\section{INTRODUCTION}

In this paper we study a homogenization problem related to the micromagnetic energy functional, precisely we seek to characterize the energy of a homogeneous ferromagnetic material whose overall response is that of a periodic material when the size of the periodicity cell tends to zero.

In the Landau and Lifschitz theory of Micromagnetics (see $[5,15]$ ), the observable states of a rigid ferromagnetic material occupying a configuration $\Omega \subset \mathbb{R}^{3}$, subject to a given external magnetic field $h_{e}$, correspond to minimizers of the total energy

$$
E_{\alpha}(m):=\frac{\alpha^{2}}{2} \int_{\Omega}|\nabla m|^{2} \mathrm{~d} x+\int_{\Omega} \varphi(m) \mathrm{d} x-\int_{\Omega} h_{e} \cdot m \mathrm{~d} x+\frac{1}{2} \int_{\mathbb{R}^{3}}\left|\nabla h_{m}\right|^{2} \mathrm{~d} x .
$$

Here the magnetization $m: \mathbb{R}^{3} \rightarrow \mathbb{R}^{3}$ represents the mass density of the macroscopic magnetic moment of the body and is subject to the constraint

$$
|m(x)|=M_{T} \chi_{\Omega}(x) \text { for a.e. } x \in \mathbb{R}^{3},
$$

where $T$ is the temperature and $M_{T}$ is the saturation magnetization, a function of the temperature and of the specific material, that is zero above the Curie point (see $[5,15]$ ). The condition $(1.1)$ ensures that the body is always saturated. Here we will assume that the temperature is held fixed, so $M_{T}$ is constant and, as it is usual, without loss of generality we will fix $M_{T}=1$.

\footnotetext{
Keywords and phrases. Micromagnetics, homogenization, $\Gamma$-convergence.

1 Dipartimento di Matematica e Applicazioni "R. Caccioppoli", Universitá degli Studi di Napoli "Federico II", Italy.

2 Département de Mathématique, E.P.F.L., Lausanne, Suisse;

e-mail: giovanni.pisante@epfl.ch \& pisante@unina.it
} 
The induced magnetic field $h_{m}: \mathbb{R}^{3} \rightarrow \mathbb{R}^{3}$ is a function related to $m$ through the Maxwell equations for magnetostatic

$$
A\left(m, h_{m}\right):=\left(\begin{array}{c}
\operatorname{div}\left(m+h_{m}\right) \\
\operatorname{curl} h_{m}
\end{array}\right)=0 \text { in } \mathbb{R}^{3} .
$$

The anisotropy energy density $\varphi: S^{2} \rightarrow \mathbb{R}$, where $S^{2}$ is the unit sphere of $\mathbb{R}^{3}$, is a non negative, even, continuous function, vanishing only on a finite set of unit vectors, called easy axes.

The four terms in $E_{\alpha}$ are designated by exchange energy, anisotropy energy, interaction energy and magnetostatic energy (for further details on the precise physical meaning of these terms see for example $[2,5,9,13]$ ).

The existence of absolute minimizers for $E_{\alpha}$ has been established by Visintin (see [18]) using the Direct Method of the Calculus of Variations, and their structure strongly depends on the competition between the different terms in the energy functional. Some work on local minimizers can be found in [2] (for further related work we refer to $[1,7,9-11,14])$. A thorough study of the limiting behavior of minimizers of $E_{\alpha}$ was carried out by De Simone in $[9,10]$, where, in particular, he showed that when the volume of the body becomes infinite then the limit behavior of minimizers of $E_{\alpha}$ is represented by the minimizers and minimizing sequences of the functional

$$
E(m):=\int_{\Omega} \varphi(m(x)) \mathrm{d} x-\int_{\Omega} h_{e}(x) \cdot m(x) \mathrm{d} x+\frac{1}{2} \int_{\mathbb{R}^{3}}\left|\nabla h_{m}(x)\right|^{2} \mathrm{~d} x .
$$

Thus this model is a good approximation for large ferromagnetic bodies.

The functional defined by (1.3), usually called no exchange energy was proposed by James and Kinderlehrer [14], where they showed that $E$ is no longer semi-continuous with respect to the natural topology for $m$, the $L^{\infty}\left(\Omega ; \mathbb{R}^{3}\right)$ weak-* topology (minimizing sequence may develop oscillations and existence of minimizers is no longer guaranteed).

The integral representation formula for the relaxed functional of $E$ was obtained by Tartar (see $[16,17])$ combining Young measures and H-measures, then, using different arguments by De Simone (see [9]). In this direction goes also the work of Fonseca and Leoni [13], where they prove a relaxation result for a general class of functionals which includes $E$, using an argument based on Young measures and the blow-up method. They recover as corollaries the results of $[9,16,17]$.

Our interest in studying the homogenization of a class of periodic micromagnetic energy functionals starts from a paper of Braides, Fonseca and Leoni (see [4]) where they give an integral representation formula for the relaxed energy and of $\Gamma$-limits of integral functionals depending on fields which are constrained to satisfy a system of first order linear partial differential equations with constant rank. Again the main idea is based on Young measures and on the blow-up method in a general setting of A-quasi-convexity.

Magnetostatic equations (see (1.2)) are a special case of constant rank operators, and so we may try to apply the general result obtained in [4] to the Maxwell operator to recover the relaxation result as the homogenization result. However this is not possible, i.e. we cannot apply directly the theory of A-quasi-convexity, since the general result holds in the case of bounded domains and when no constraints of the type (1.1) are present.

For this reason, in the Magnetostatic framework we must develop an ad hoc procedure and, indeed, this is what is done in [13] concerning the relaxation. We study the homogenization problem using a similar idea, looking at it as a $\Gamma$-convergence problem (see $[3,8]$ ).

In what follows $\Omega \subset \mathbb{R}^{3}$ is an open bounded set with Lipschitz boundary, and we define the set $\mathcal{M}$ of admissible magnetizations as

$$
\mathcal{M}:=\left\{m: \mathbb{R}^{3} \rightarrow \mathbb{R}^{3} \text { measurable }:|m(x)|=\chi_{\Omega}(x) \text { a.e. in } \mathbb{R}^{3}\right\} .
$$

Consider the anisotropy energy density

$$
\phi: \mathbb{R}^{3} \times \mathbb{R}^{3} \times\left(S^{2} \cup\{0\}\right) \rightarrow[0, \infty)
$$

such that

$$
0 \leq \phi(x, y, m) \leq a(x)
$$


with $\phi(x, y, m) Q$-periodic in the second variable, that is $\phi\left(x, y+e_{i}, m\right)=\phi(x, y, m)$ for every $i=1,2,3$ where $e_{i}$ are the elements of the canonical basis of $\mathbb{R}^{3}$, and $a \in L^{1}\left(\mathbb{R}^{3}\right)$. Let $\varepsilon>0$ and consider, for $m \in \mathcal{M}$, the family of no-exchange energy functionals

$$
\mathcal{E}_{\varepsilon}(m)=\int_{\Omega} \phi\left(x, \frac{x}{\varepsilon}, m(x)\right) \mathrm{d} x-\int_{\Omega} h_{e}(x) \cdot m(x) \mathrm{d} x+\frac{1}{2} \int_{\mathbb{R}^{3}}|\nabla u(x)|^{2} \mathrm{~d} x
$$

where $u \in H^{1}\left(\mathbb{R}^{3}\right)$ is the unique solution of Maxwell equation (see [14])

$$
\Delta u+\operatorname{div} m=0 \text { in } \mathbb{R}^{3} .
$$

In this formulation the domain $\Omega$ represents the body configuration and the function $m$ its magnetization. Moreover, we note that in the notation already introduced for the magnetostatic equations we have that $(m, \nabla u) \in \operatorname{Ker} A$ where $A$ is the constant rank operator defined by (1.2).

In order to give the definition of $\Gamma$-limit for the class of functionals $\mathcal{E}_{\varepsilon}$ we need to introduce the domain of definition of the $\Gamma$-limit functional, $\mathcal{M}^{*}$, defined by

$$
\mathcal{M}^{*}:=\left\{m: \mathbb{R}^{3} \rightarrow \mathbb{R}^{3} \text { measurable }:|m(x)| \leq \chi_{\Omega}(x) \text { a.e. in } \mathbb{R}^{3}\right\} .
$$

Let $\varepsilon_{n} \rightarrow 0^{+}$. We say that the functional $\mathcal{E}(m): \mathcal{M}^{*} \rightarrow \overline{\mathbb{R}}$ is the $\Gamma-\lim \inf [$ resp. $\Gamma-\lim$ sup] of the sequence of functionals $\mathcal{E}_{\varepsilon_{n}}$ with respect to the $L^{\infty}$-weak* convergence if for every $m \in \mathcal{M}^{*}$

$$
\mathcal{E}(m):=\inf \left\{\liminf _{n \rightarrow \infty}\left[\text { resp. } \limsup _{n \rightarrow \infty}\right] \mathcal{E}_{\mathcal{E}_{n}}\left(m_{n}\right): m_{n} \in \mathcal{M}, m_{n} \stackrel{*}{\rightarrow} m \text { in } L^{\infty}(\Omega)\right\},
$$

and we write

$$
\mathcal{E}=\Gamma-\liminf _{n \rightarrow \infty} \mathcal{E}_{\varepsilon_{n}}\left[\text { resp. } \limsup _{n \rightarrow \infty} \mathcal{E}_{\varepsilon_{n}}\right] .
$$

We say that the sequence $\mathcal{E}_{\mathcal{E}_{n}} \Gamma$-converges to $\mathcal{E}$ if the $\Gamma-\liminf$ and $\Gamma-\lim$ sup coincide and we write $\mathcal{E}=\Gamma-\lim \mathcal{E}_{\varepsilon_{n}}$.

The functional $\mathcal{E}$ is said to be the $\Gamma-\lim \inf \left[\right.$ resp. $\Gamma-\lim \sup$ ] of the family of functionals $\mathcal{E}_{\varepsilon}$ with respect to the $L^{\infty}$-weak* convergence if for every sequence $\varepsilon_{n} \rightarrow 0^{+}$we have that

$$
\mathcal{E}=\Gamma-\liminf _{n \rightarrow \infty} \mathcal{E}_{\varepsilon_{n}}\left[\text { resp. } \limsup _{n \rightarrow \infty} \mathcal{E}_{\varepsilon_{n}}\right]
$$

and we write

$$
\mathcal{E}=\Gamma-\liminf _{\varepsilon \rightarrow 0} \mathcal{E}_{\varepsilon}\left[\text { resp. } \limsup _{\varepsilon \rightarrow 0} \mathcal{E}_{\varepsilon}\right]
$$

Finally, we say that $\mathcal{E}$ is the $\Gamma$-limit of the family $\mathcal{E}_{\mathcal{E}}$, and we write

$$
\mathcal{E}=\Gamma-\lim _{\varepsilon \rightarrow 0} \mathcal{E}_{\varepsilon},
$$

if $\Gamma-\lim \inf$ and $\Gamma-\lim \sup$ coincide.

The main result of the paper is given by the following theorem:

Theorem 1.1. If the anisotropy energy $\phi$ does not depend on $x$, then the $\Gamma$-limit $\mathcal{E}_{\text {hom }}$ of $\mathcal{E}_{\varepsilon}$ exists and we have

$$
\mathcal{E}_{\text {hom }}(m)=\int_{\Omega} f_{\text {hom }}(m(x), \nabla u(x)) \mathrm{d} x-\int_{\Omega} h_{e}(x) \cdot m(x) \mathrm{d} x+\frac{1}{2} \int_{\mathbb{R}^{3} \backslash \bar{\Omega}}|\nabla u(x)|^{2} \mathrm{~d} x
$$


for all $m \in \mathcal{M}^{*}, u \in H^{1}(\Omega)$ such that $\operatorname{div}(m+\nabla u)=0$ in $\mathbb{R}^{3}$. The energy density $f_{\text {hom }}: \bar{B} \times \mathbb{R}^{3} \rightarrow \mathbb{R}$, with $\bar{B}$ the unit ball of $\mathbb{R}^{3}$, is defined by

$$
f_{\text {hom }}(\mu, h):=\inf _{S_{\mu}}\left\{\liminf _{k \rightarrow \infty} \int_{Q} \phi\left(k y, M_{k}(y)\right)+\frac{1}{2}\left|h+\nabla U_{k}(y)\right|^{2} \mathrm{~d} y\right\}
$$

where $Q$ is the unit cube and

$$
S_{\mu}:=\left\{\begin{array}{c}
\left\{M_{k}, U_{k}\right\} \in L^{\infty}\left(Q, \mathbb{R}^{3}\right) \times\left(H_{0}^{1}(Q)\right):\left|M_{k}\right|=\chi_{Q}, \\
M_{k} \stackrel{*}{\rightarrow} \mu \text { in } L^{\infty}\left(Q, \mathbb{R}^{3}\right), \operatorname{div} M_{k}+\Delta U_{k}=0 \text { in } Q
\end{array}\right\} .
$$

We give here a simple outline of the proof. We can obtain this result basically in three steps. First we simplify the functionals $\mathcal{E}_{\varepsilon}$, showing that the non local part of the magnetostatic energy

$$
\frac{1}{2} \int_{\mathbb{R}^{3} \backslash \bar{\Omega}}|\nabla u(x)|^{2} \mathrm{~d} x
$$

and the interaction energy

$$
\int_{\Omega} h_{e}(x) \cdot m(x) \mathrm{d} x
$$

are continuous perturbations for our $\Gamma$-limit ( $c f$. Sect. 2). So we can restrict our study to the simpler class of functionals

$$
\mathcal{F}_{\varepsilon}(m):=\int_{\Omega}\left(\phi\left(x, \frac{x}{\varepsilon}, m\right)+\frac{1}{2}|\nabla u|^{2}\right) \mathrm{d} x .
$$

Then we follow the usual procedure, that is to work with a particular subsequence, $\mathcal{F}_{\varepsilon_{k}}$, with nice properties, whose existence is ensured by Lemma 3.3, to identify its $\Gamma$-limit and then to show that it is the $\Gamma$-limit which we are looking for.

The second step consists in proving the existence of an integral representation for the $\Gamma-\lim \inf \mathcal{F}_{\varepsilon_{k}}$, using the De Giorgi-Letta lemma (see [3] or [8]). More precisely we define a localization of $\mathcal{F}_{\varepsilon_{k}}$ as follows

$$
\mathcal{F}_{\varepsilon_{k}}(m ; D):=\int_{D}\left(\phi\left(x, \frac{x}{\varepsilon_{k}}, m\right)+\frac{1}{2}|\nabla u|^{2}\right) \mathrm{d} x
$$

and we prove that the $\Gamma-\liminf \mathcal{F}_{\varepsilon_{k}}(m, \cdot)$, as a set function, is the trace of a Radon measures absolutely continuous with respect to Lebesgue measure on $\mathbb{R}^{3}$ ( $c f$. Lem. 3.5).

Finally we identify in $f_{\text {hom }}(m(x), \nabla u(x))$ the Radon-Nikodym derivative of $\Gamma-\liminf \mathcal{F}_{\varepsilon_{k}}(m, \cdot)$ in $x$ when the anisotropy energy density is autonomous (cf. Prop. 3.7).

Remark 1.2. We should point out that in recovering the homogenization result of Theorem 1.1, the hypothesis of autonomy of the anisotropy energy density (i.e. that $\phi$ does not depend on the position $x$ ) is not necessary to prove the existence of the integral representation of $\mathcal{E}_{\text {hom }}(m)$. Nevertheless this assumption makes more clear and not burned by too many technicalities the computation of the homogeneous energy density $f_{\text {hom }}$.

\section{Preliminaries}

In this section we first recall some results on the convergence of the solutions of the Maxwell equations, then we make some remarks on the class of functionals $\left\{\mathcal{E}_{\varepsilon}\right\}$ in order to simplify the further calculations.

The proofs of the following lemmata can be founded in [13]. 
Lemma 2.1. Let $m \in \mathcal{M}^{*}$ and consider a sequence $\left\{m_{k}\right\} \subset \mathcal{M}^{*}$ such that $m_{k} \stackrel{*}{\rightarrow} m$ in $L^{\infty}\left(\Omega ; \mathbb{R}^{3}\right)$. Let $u, u_{k} \in H^{1}\left(\mathbb{R}^{3}\right)$ be such that $\Delta u_{k}+\operatorname{div} m_{k}=\Delta u+\operatorname{div} m=0$ in $\mathbb{R}^{3}$. Then $\left\{\nabla u_{k}\right\}$ is 2 -equi-integrable over $\mathbb{R}^{3}$, $u_{k} \rightarrow u$ in $H_{l o c}^{1}\left(\mathbb{R}^{3}\right), u_{k} \rightarrow u$ in $L_{l o c}^{\infty}\left(\mathbb{R}^{3}\right)$, and

$$
\lim _{k \rightarrow \infty}\left\|u_{k}-u\right\|_{L^{2^{*}\left(\mathbb{R}^{3}\right)}}=\lim _{k \rightarrow \infty}\left\|\nabla u_{k}-\nabla u\right\|_{L^{2}\left(\mathbb{R}^{3} \backslash \bar{\Omega}\right)}=0 .
$$

Lemma 2.2. Let $D$ be an open bounded set of $\mathbb{R}^{3}$ and consider a sequence

$$
\left\{\left(m_{k}, u_{k}\right)\right\} \subset L^{\infty}(D ; \bar{B}) \times\left(\phi_{k}+H_{0}^{1}(D)\right)
$$

such that $\Delta u_{k}+\operatorname{div} m_{k}=0$ in $D$, where $\phi_{k} \in H^{1}(D)$. If $\phi_{k} \rightarrow \phi$ in $H^{1}(D)$ and $m_{k} \stackrel{*}{\rightarrow} m$ in $L^{\infty}\left(\Omega ; \mathbb{R}^{3}\right)$ then $u_{k} \rightarrow u$ in $H^{1}(D)$, where $u \in \phi+H_{0}^{1}(D)$ is the unique solution of $\Delta u+\operatorname{div} m=0$ in D. Moreover, if $\left\{\nabla \phi_{k}\right\}$ is 2-equi-integrable over $D$ then $\left\{\nabla u_{k}\right\}$ is also 2-equi-integrable over D. Finally, if in addition we also assume that $m_{k} \rightarrow m$ strongly in $L^{2}\left(\Omega ; \mathbb{R}^{3}\right)$ then $u_{k} \rightarrow u$ strongly in $H^{1}(D)$.

Now we recall that by definition of $\Gamma$-convergence (see $[3,8]$ ) if $F$ is the $\Gamma$-limit of a sequence of functionals $F_{j}$ in the metric space $(X, d)$ and $G$ is a functional continuous with respect to the topology of $(X, d)$, then

$$
F+G=\Gamma-\lim _{j}\left(F_{j}+G\right)
$$

and $G$ is called continuous perturbation of the $\Gamma$-limit.

Using this terminology, we observe that the interaction energy

$$
\int_{\Omega} h_{e}(x) \cdot m(x) \mathrm{d} x
$$

is, in fact, a continuous perturbation of our $\Gamma-\lim \mathcal{E}_{\varepsilon}$. Indeed we are working in the unit ball $B_{\infty}$ of $L^{\infty}\left(\Omega ; \mathbb{R}^{3}\right)$ endowed with the weak* topology, which is metrizable, let's call $d_{\infty}^{*}$ its metric. Moreover if $h_{e} \in L_{\text {loc }}^{1}\left(\mathbb{R}^{3}\right)$ then the interaction energy $(2.1)$ is, by definition, continuous with respect to the topology of $\left(B_{\infty}, d_{\infty}^{*}\right)$, so in order to calculate the $\Gamma-\lim \mathcal{E}_{\varepsilon}=\Gamma\left(d_{\infty}^{*}\right)-\lim \mathcal{E}_{\varepsilon}$ it is sufficient to study the $\Gamma\left(d_{\infty}^{*}\right)$-limit of the family of functionals $\widetilde{\mathcal{E}}_{\varepsilon}$ defined by

$$
\widetilde{\mathcal{E}}_{\varepsilon}(m)=\int_{\Omega} \phi\left(x, \frac{x}{\varepsilon}, m\right) \mathrm{d} x+\int_{\mathbb{R}^{3}} \frac{1}{2}|\nabla u|^{2} \mathrm{~d} x .
$$

We will see in the next section that the first step in the study of the $\Gamma$-limit of a class of integral functionals is the localization. In order to well define this localization we need to rewrite our functionals in a nicer form, since the non local term (the magnetostatic energy) a priori can give us some troubles. Then we split the functionals $\widetilde{\mathcal{E}}_{\varepsilon}$ in a local part and in a non local part as follows

$$
\widetilde{\mathcal{E}}_{\varepsilon}(m)=\int_{\Omega}\left(\phi\left(x, \frac{x}{\varepsilon}, m\right)+\frac{1}{2}|\nabla u|^{2}\right) \mathrm{d} x+\int_{\mathbb{R}^{3} \backslash \bar{\Omega}} \frac{1}{2}|\nabla u|^{2} \mathrm{~d} x .
$$

We observe that by Lemma 2.1 the nonlocal part is continuous with respect to the topology used for the $\Gamma$-limit and if we have $m_{\varepsilon} \stackrel{*}{\rightarrow} m$ in $L^{\infty}\left(\Omega, \mathbb{R}^{3}\right)$ then $\left\{\nabla u_{\varepsilon}\right\}$ is 2-equi-integrable over $\mathbb{R}^{3}$ and $\nabla u_{\varepsilon} \rightarrow \nabla u$ in $L^{2}\left(\mathbb{R}^{3} \backslash \bar{\Omega}\right)$ with $\Delta u_{\varepsilon}+\operatorname{div} m_{\varepsilon}=\Delta u+\operatorname{div} m=0$ in $\mathbb{R}^{3}$.

This remark allows us to restrict our study to the homogenization of the class of local functionals $\left\{\mathcal{F}_{\varepsilon}\right\}$ defined by

$$
\mathcal{F}_{\varepsilon}(m):=\int_{\Omega}\left(\phi\left(x, \frac{x}{\varepsilon}, m\right)+\frac{1}{2}|\nabla u|^{2}\right) \mathrm{d} x .
$$

Finally we recall a classical result from $\Gamma$-convergence that we will need in the sequel (see [3] for a proof). 
Proposition 2.3. Let $(X, d)$ be a separable metric space and let $f_{n}: X \rightarrow[-\infty, \infty]$. Then

(i) there exists an increasing sequence of integers $\left\{n_{k}\right\}$ such that

$$
\Gamma-\lim _{k \rightarrow \infty} f_{n_{k}}(x) \text { exists for all } x \in X
$$

(ii) moreover

$$
f_{\infty}=\Gamma(d)-\lim _{n \rightarrow \infty} f_{n}
$$

if and only if for every subsequence $\left\{f_{n_{k}}\right\}$ there exists a further subsequence $\left\{f_{n_{k_{j}}}\right\}$ which $\Gamma(d)$-converges to $f_{\infty}$.

\section{Homogenization}

In this section we study the homogenization of the class $\left\{\mathcal{F}_{\varepsilon}\right\}$, that is we prove the existence of the $\Gamma-$ $\lim _{\varepsilon \rightarrow 0} \mathcal{F}_{\varepsilon}$ and we give an integral representation for it.

We start introducing a localization of $\mathcal{F}_{\varepsilon}$. Let $\mathcal{O}(\Omega)$ denote the class of all open subsets of $\Omega$. For every $\varepsilon>0, D \in \mathcal{O}(\Omega)$ and $m \in \mathcal{M}$ we consider the functional $\mathcal{F}_{\varepsilon}:(\mathcal{M} \times \mathcal{O}(\Omega)) \rightarrow[0, \infty)$ defined by

$$
\mathcal{F}_{\varepsilon}(m ; D):=\int_{D}\left(\phi\left(x, \frac{x}{\varepsilon}, m\right)+\frac{1}{2}|\nabla u|^{2}\right) \mathrm{d} x .
$$

Let $\varepsilon_{k} \rightarrow 0^{+}$. We can write, by definition of $\mathcal{F}_{\varepsilon}$ and of the $\Gamma-\lim$ inf, that for every $m \in \mathcal{M}^{*}$

$$
\Gamma-\liminf \mathcal{F}_{\varepsilon_{k}}(m ; D):=\inf \left\{\liminf _{k \rightarrow \infty} \int_{D}\left(\phi\left(x, \frac{x}{\varepsilon_{k}}, m_{k}\right)+\frac{1}{2}\left|\nabla u_{k}\right|^{2}\right) \mathrm{d} x\right\}
$$

where the infimum is taken for $\left\{m_{k}, u_{k}\right\} \in \mathbf{M}_{\mathbf{D}}$ with

$$
\mathbf{M}_{\mathbf{D}}:=\left\{\begin{array}{c}
\left\{m_{k}, u_{k}\right\} \subset \mathcal{M}^{*} \times H^{1}\left(\mathbb{R}^{3}\right):\left|m_{k}\right|=1 \text { in } D, m_{k}=m \text { in } \mathbb{R}^{3} \backslash D, \\
m_{k} \stackrel{*}{\rightarrow} m \text { in } L^{\infty}\left(D ; \mathbb{R}^{3}\right), \Delta u_{k}+\operatorname{div} m_{k}=0 \text { in } \mathbb{R}^{3}
\end{array}\right\} .
$$

In the following lemma we prove that in the definition of $\Gamma-\lim \inf (3.1)$ we can change the space where we minimize, in order to use Sobolev functions defined on $D$ with fixed boundary values, instead of functions in $H^{1}\left(\mathbb{R}^{3}\right)$, and this will simplify the further calculations.

Lemma 3.1. For any $D \in \mathcal{O}(\Omega)$ and $m \in \mathcal{M}^{*}$ we have

$$
\Gamma-\liminf \mathcal{F}_{\varepsilon_{k}}(m ; D)=\mathcal{F}_{0}(m ; D)
$$

where

with

$$
\mathcal{F}_{0}(m ; D):=\inf _{\left\{m_{k}, u_{k}\right\} \in \mathbf{M}_{\mathbf{D}}^{\prime}}\left\{\liminf _{k \rightarrow \infty} \int_{D}\left(\phi\left(x, \frac{x}{\varepsilon_{k}}, m_{k}\right)+\frac{1}{2}\left|\nabla u_{k}\right|^{2}\right) \mathrm{d} x\right\},
$$

$$
\mathbf{M}_{\mathbf{D}}^{\prime}:=\left\{\begin{array}{c}
\left\{m_{k}, u_{k}\right\} \subset L^{\infty}\left(D, S^{2}\right) \times\left(u+H_{0}^{1}(D)\right) \\
m_{k} \stackrel{*}{\rightarrow} m \text { in } L^{\infty}\left(D ; \mathbb{R}^{3}\right), \Delta u_{k}+\operatorname{div} m_{k}=0 \text { in } D
\end{array}\right\} .
$$

Remark 3.2. Note that $\mathbf{M}_{\mathbf{D}}^{\prime}$ is well defined since it is well known that for any $D \in \mathcal{O}(\Omega), u \in H^{1}(D)$ and $m \in L^{2}\left(D ; \mathbb{R}^{3}\right)$, there exists a unique solution of the generalized Dirichlet problem

$$
\left\{\begin{array}{l}
\Delta v+\operatorname{div} m=0 \text { in } D \\
v-u \in H_{0}^{1}(D) .
\end{array}\right.
$$


Proof of Lemma 3.1. Let $\left\{m_{k}, u_{k}\right\} \in \mathbf{M}_{\mathbf{D}}^{\prime}$ such that

$$
\lim _{k \rightarrow \infty} \int_{D} \phi\left(x, \frac{x}{\varepsilon_{k}}, m_{k}\right)+\frac{1}{2}\left|\nabla u_{k}\right|^{2} \mathrm{~d} x=\liminf _{k \rightarrow \infty} \int_{D} \phi\left(x, \frac{x}{\varepsilon_{k}}, m_{k}\right)+\frac{1}{2}\left|\nabla u_{k}\right|^{2} \mathrm{~d} x
$$

extend $m_{k}$ to be $m$ outside $D$ and let $U_{k} \in H^{1}\left(\mathbb{R}^{3}\right)$ be the unique solution of $\Delta U_{k}+\operatorname{div} m_{k}=0$ in $\mathbb{R}^{3}$, then we have $\left\{m_{k}, U_{k}\right\} \in \mathbf{M}_{\mathbf{D}}$. Define $v_{k}:=u_{k}-U_{k}$ and $\psi_{k}:=u-U_{k}$. Then

$$
\left\{\begin{array}{l}
\Delta v_{k}=0 \text { on } D \\
v_{k} \in \psi_{k}+H_{0}^{1}(D)
\end{array}\right.
$$

since $v_{k}=\psi_{k}+\left(u_{k}-u\right)$ and $u_{k} \in u+H_{0}^{1}(D)$. Moreover $\Delta \psi_{k}=-\operatorname{div}\left(m_{k}-m\right)$ in $\mathbb{R}^{3}$ and since $m_{k}-m \stackrel{*}{\rightarrow} 0$ in $L^{\infty}\left(D ; \mathbb{R}^{3}\right)$, by Lemma 2.1 we have $\psi_{k} \rightarrow 0$ in $H_{\text {loc }}^{1}\left(\mathbb{R}^{3}\right)$ and $\left\{\nabla \phi_{k}\right\}$ is 2-equi-integrable over $\mathbb{R}^{3}$. Now using Lemma 2.2 and $(3.2)$ we can say that $v_{k} \rightarrow 0$ in $H^{1}(D)$, i.e. $\lim _{k \rightarrow \infty}\left\|u_{k}-U_{k}\right\|_{H^{1}(D)}=0$, Moreover, we observe that, by Lemma 2.2, $\left\{\nabla u_{k}\right\}$ is 2-equi-integrable on $D$ and weakly convergent in $H^{1}(D)$ (it is sufficient to consider $u_{k}$ as the solution of $\Delta u_{k}+\operatorname{div} m_{k}=0$ in $D$ with $\left.u_{k} \in u+H_{0}^{1}(D)\right)$. Then

$$
\begin{aligned}
\Gamma-\liminf \mathcal{F}_{\varepsilon_{k}}(m ; D) \leq & \liminf _{k \rightarrow \infty} \int_{D} \phi\left(x, \frac{x}{\varepsilon_{k}}, m_{k}\right)+\frac{1}{2}\left|\nabla U_{k}\right|^{2} \mathrm{~d} x \\
\leq & \liminf _{k \rightarrow \infty} \int_{D} \phi\left(x, \frac{x}{\varepsilon_{k}}, m_{k}\right)+\frac{1}{2}\left|\nabla u_{k}\right|^{2} \mathrm{~d} x \\
& +\lim _{k \rightarrow \infty} \int_{D} \frac{1}{2}\left|\nabla u_{k}-\nabla U_{k}\right|^{2} \mathrm{~d} x \\
& +\lim _{k \rightarrow \infty} \int_{D}\left|\nabla u_{k}\right|\left|\nabla u_{k}-\nabla U_{k}\right| \mathrm{d} x \\
= & \lim _{k \rightarrow \infty} \int_{D} \phi\left(x, \frac{x}{\varepsilon_{k}}, m_{k}\right)+\frac{1}{2}\left|\nabla u_{k}\right|^{2} \mathrm{~d} x .
\end{aligned}
$$

Taking the infimum on $\mathbf{M}_{\mathbf{D}}$ we have

$$
\Gamma-\liminf \mathcal{F}_{\varepsilon_{k}}(m ; D) \leq \mathcal{F}_{0}(m ; D)
$$

The opposite inequality follows taking $\left\{m_{k}, U_{k}\right\} \in \mathbf{M}_{\mathbf{D}}$ such that

$$
\lim _{k \rightarrow \infty} \int_{D} \phi\left(x, \frac{x}{\varepsilon_{k}}, m_{k}\right)+\frac{1}{2}\left|\nabla U_{k}\right|^{2} \mathrm{~d} x=\liminf _{k \rightarrow \infty} \int_{D} \phi\left(x, \frac{x}{\varepsilon_{k}}, m_{k}\right)+\frac{1}{2}\left|\nabla U_{k}\right|^{2} \mathrm{~d} x
$$

letting $u_{k} \in u+H_{0}^{1}(D)$ be the unique solution of $\Delta u_{k}+\operatorname{div} m_{k}=0$ in $D$ and using the same argument as above.

In the next lemma, we use the first part of Proposition 2.3 to prove that from each sequence $\varepsilon_{n} \rightarrow 0^{+}$we can extract a subsequence that admits the $\Gamma$-limit for a dense family of open sets of $\mathcal{O}(\Omega)$. This result allows us to follow the usual procedure, that is to work with a particular subsequence with nice properties, to identify its $\Gamma$-limit and then to show that it is the $\Gamma$-limit which we are looking for. 
Lemma 3.3. Let $\varepsilon_{n} \rightarrow 0$ and let $\mathcal{R}(\Omega)$ be the family of all finite unions of open cubes contained in $\Omega$ and with vertices in $\mathbb{Q}^{3}$. Then there exists a subsequence $\left\{\varepsilon_{n_{k}}\right\}$ of $\left\{\varepsilon_{n}\right\}$ such that the $\Gamma$ - limit

$$
\Gamma-\lim _{k \rightarrow \infty} \mathcal{F}_{\varepsilon_{n_{k}}}(m ; R)
$$

exists for all $m \in \mathcal{M}^{*}$ and for all $R \in \mathcal{R}(\Omega)$.

Proof. Fix $R \in \mathcal{R}(\Omega)$. For simplicity set $\mathcal{F}_{\varepsilon_{n}}=\mathcal{F}_{n}$. We can consider $\mathcal{F}_{n}$ as a functional defined for $(m, h) \in$ $\left\{L^{\infty}\left(\mathbb{R}^{3} ; S^{2}\right) \times L^{2}\left(\mathbb{R}^{3} ; \mathbb{R}^{3}\right)\right\} \cap \operatorname{Ker}(A)$, as

$$
\mathcal{F}_{n}(m ; h)=\mathcal{F}_{n}(m ; h ; R)=\int_{R} \phi\left(x, \frac{x}{\varepsilon_{n}}, m\right)+\frac{1}{2}|h|^{2} \mathrm{~d} x,
$$

where $A$ is the operator defined by (1.2)

$$
A(m, h):=\left(\begin{array}{c}
\operatorname{div}(m+h) \\
\operatorname{curl} h
\end{array}\right)
$$

Indeed, if $(m, h) \in\left\{L^{\infty}\left(\mathbb{R}^{3} ; S^{2}\right) \times L^{2}\left(\mathbb{R}^{3} ; \mathbb{R}^{3}\right)\right\} \cap \operatorname{Ker}(A)$ then there exists $u \in H^{1}\left(\mathbb{R}^{3}\right)$, such that $h=\nabla u$ and $\Delta u+\operatorname{div} m=0$ in $\mathbb{R}^{3}$.

Let $B_{\infty}$ denote the closed unit ball of $L^{\infty}\left(R ; \mathbb{R}^{3}\right)$ and for each $l \in \mathbb{N}$ consider

$$
l B_{2}=\left\{v \in L^{2}\left(R ; \mathbb{R}^{3}\right):\|v\|_{L^{2}} \leq l\right\} .
$$

We observe that the space $B_{\infty} \times l B_{2}$ endowed with the weak*-convergence in $L^{\infty}$ and the weak-convergence in $L^{2}$ is metrizable. Indeed $B_{\infty}$ with the weak*-topology is metrizable since it is compact and separable, and $l B_{2}$ with the weak-topology is metrizable, since the dual space of $L^{2}\left(R ; \mathbb{R}^{3}\right)$ is separable. Let's denote by $d_{l}$ the metric on $B_{\infty} \times l B_{2}$.

Consider $l=1$ and apply Proposition 2.3 to the sequence of functionals $\left\{\mathcal{F}_{n}(\cdot ; \cdot ; R)\right\}$ restricted to $\left\{B_{\infty} \times\right.$ $\left.B_{2} \cap \operatorname{Ker}(A), d_{1}\right\}$. Then we can find an increasing sequence of integers $\left\{n_{j}^{1}\right\}$ such that

$$
\Gamma\left(d_{1}\right)-\lim _{j \rightarrow \infty} \mathcal{F}_{n_{j}^{1}}(v ; w ; R)
$$

exists for all $(v, w) \in\left\{B_{\infty} \times B_{2}\right\} \cap \operatorname{Ker}(A)$. Now we proceed recursively, so that given $l \in \mathbb{N}$ we apply the Proposition 2.3 to the sequence $\left\{\mathcal{F}_{n_{j}^{l-1}}(\cdot ; \cdot ; R)\right\}$ restricted to $\left\{B_{\infty} \times l B_{2} \cap \operatorname{Ker}(A), d_{l}\right\}$ to obtain a subsequence $\left\{n_{j}^{l}\right\}$ of $\left\{n_{j}^{l-1}\right\}$ such that

$$
\Gamma\left(d_{l}\right)-\lim _{j \rightarrow \infty} \mathcal{F}_{n_{j}^{l}}(v ; w ; R)
$$

exists for all $(v, w) \in\left\{B_{\infty} \times l B_{2}\right\} \cap \operatorname{Ker}(A)$. Let $n_{k}:=n_{k}^{k}$. Since $\left\{n_{k}\right\}$ is a subsequence of all $\left\{n_{j}^{l}\right\}$ we have that for each $l \in \mathbb{N}$

$$
\Gamma\left(d_{l}\right)-\lim _{k \rightarrow \infty} \mathcal{F}_{n_{k}}(v ; w ; R)
$$

exists for all $(v, w) \in\left\{B_{\infty} \times l B_{2}\right\} \cap \operatorname{Ker}(A)$.

We claim that the $\Gamma$-limit

$$
\Gamma-\lim _{k \rightarrow 0} \mathcal{F}_{n_{k}}(v ; w ; R)
$$

exists for all $(v, w) \in\left\{B_{\infty} \times L_{2}\left(R ; \mathbb{R}^{3}\right)\right\} \cap \operatorname{Ker}(A)$. Indeed assume by contradiction that this is not the case. Then there exists $(v, w) \in\left\{B_{\infty} \times L_{2}\left(R ; \mathbb{R}^{3}\right)\right\} \cap \operatorname{Ker}(A)$ for which

$$
\mathcal{F}^{-}(v ; w):=\Gamma-\liminf _{k \rightarrow \infty} \mathcal{F}_{n_{k}}(v ; w ; R)<\mathcal{F}^{+}(v ; w):=\Gamma-\limsup _{k \rightarrow \infty} \mathcal{F}_{n_{k}}(v ; w ; R) .
$$


Let $\left(v_{k}, w_{k}\right) \in\left\{B_{\infty} \times L_{2}\left(R ; \mathbb{R}^{3}\right)\right\} \cap \operatorname{Ker}(A)$ such that $v_{k} \stackrel{*}{\rightarrow} v$ in $L^{\infty}\left(R ; \mathbb{R}^{3}\right)$ and $w_{k} \rightarrow w$ in $L^{2}\left(R ; \mathbb{R}^{2}\right)$ and

$$
\liminf _{k \rightarrow \infty} \mathcal{F}_{n_{k}}\left(v_{k} ; w_{k}, R\right)=\mathcal{F}^{-}(v ; w) .
$$

Since $w_{k} \rightarrow w$ in $L^{2}\left(R ; \mathbb{R}^{2}\right)$ we may find an integer $l_{0}$ such that $\left(v_{k}, w_{k}\right),(v, w) \in\left\{B_{\infty} \times l_{0} B_{2}\right\} \cap \operatorname{Ker}(A)$ for all $k \in \mathbb{N}$. Consequently

$$
d_{l_{0}}\left(\left(v_{k}, w_{k}\right),(v, w)\right) \rightarrow 0 \text { as } k \rightarrow \infty,
$$

and thus

$$
\begin{aligned}
\Gamma\left(d_{l_{0}}\right)-\liminf _{k \rightarrow \infty} \mathcal{F}_{n_{k}}(v ; w ; R) & \leq \liminf _{k \rightarrow \infty} \mathcal{F}_{n_{k}}(v ; w ; R) \\
& =\mathcal{F}^{-}(v ; w)<\mathcal{F}^{+}(v ; w) \\
& \leq \Gamma\left(d_{l_{0}}\right)-\limsup _{k \rightarrow \infty} \mathcal{F}_{n_{k}}(v ; w ; R),
\end{aligned}
$$

which contradict the existence of the $\Gamma$-limit $\Gamma\left(d_{l_{0}}\right)-\lim _{k \rightarrow \infty} \mathcal{F}_{n_{k}}(v ; w ; R)$, and where we have used the fact that

$$
\begin{aligned}
& \mathcal{F}^{+}(v ; w)=\inf \left\{\limsup _{k \rightarrow \infty} \mathcal{F}_{n_{k}}\left(v_{k} ; w_{k} ; R\right):\left(v_{k}, w_{k}\right) \in B_{\infty} \times L^{2}(R) \cap \operatorname{Ker}(A),\right. \\
& \left.\left(v_{k}, w_{k}\right) \stackrel{*}{\rightarrow}, \rightarrow(v, w) \text { in } B_{\infty} \times L^{2}(R)\right\} \\
& \leq \Gamma\left(d_{l_{0}}\right)-\limsup _{k \rightarrow \infty} \mathcal{F}_{n_{k}}(v ; w ; R) \\
& =\inf \left\{\limsup _{k \rightarrow \infty} \mathcal{F}_{n_{k}}\left(v_{k} ; w_{k} ; R\right):\left(v_{k}, w_{k}\right) \in B_{\infty} \times l_{0} B_{2} \cap \operatorname{Ker}(A),\right. \\
& \left.\left(v_{k}, w_{k}\right) \stackrel{*}{\rightarrow}, \rightarrow(v, w) \text { in } B_{\infty} \times L^{2}(R)\right\} .
\end{aligned}
$$

Hence (3.3) holds. To conclude the proof it suffices to observe that since the family $\mathcal{R}(\Omega)$ is countable, with a diagonal argument it is possible to extract a further subsequence for which (3.3) holds for all $R \in \mathcal{R}(\Omega)$.

Remark 3.4. The previous proof asserts that for any given $D \in \mathcal{O}(\Omega)$ and $\varepsilon_{n} \rightarrow 0^{+}$there exists a subsequence $\left\{\varepsilon_{n_{k}}\right\}$ (depending on the particular set $D$ ) of $\left\{\varepsilon_{n}\right\}$ such that the $\Gamma$-limit

$$
\Gamma-\lim _{k \rightarrow \infty} \mathcal{F}_{\varepsilon_{n_{k}}}(m ; D)
$$

exists for all $m \in \mathcal{M}^{*}$.

Given $\varepsilon_{n} \rightarrow 0^{+}$let $\varepsilon_{n_{k}}$ as in Lemma 3.3. For any $m \in \mathcal{M}^{*}$ and $D \in \mathcal{O}(\Omega)$ set

$$
\mathcal{F}^{-}(m ; D):=\Gamma-\liminf \mathcal{F}_{\varepsilon_{n_{k}}}(m ; D) .
$$

The next step is to prove the existence of an integral representation for $\mathcal{F}^{-}(m ; D)$. In this direction goes the following lemma.

Lemma 3.5. For every $m \in \mathcal{M}^{*}, \mathcal{F}^{-}(m ; \cdot)$ is the trace of a Radon measure.

Proof. To simplify the notations in this proof we will refer to $\left\{\varepsilon_{n_{k}}\right\}$ simply as $\{\varepsilon\}$. In order to prove that $\mathcal{F}^{-}$ is the trace of a Radon measure it is suffices to prove subadditivity for nested sets, that is

$$
\mathcal{F}^{-}(m ; D) \leq \mathcal{F}^{-}(m ; D \backslash \bar{E})+\mathcal{F}^{-}(m ; B)
$$

if $E \subset \subset B \subset \subset D \subset \subset \Omega$. 
By definition of $\Gamma$-convergence and Lemma 3.1, for fixed $\eta>0$ there exist two sequences of pairs of functions $\left\{\left(m_{\varepsilon}, u_{\varepsilon}\right)\right\} \in \mathbf{M}_{\mathbf{D} \backslash \overline{\mathbf{E}}}^{\prime},\left\{\left(w_{\varepsilon}, z_{\varepsilon}\right)\right\} \in \mathbf{M}_{\mathbf{B}}^{\prime}$ such that

$$
\begin{gathered}
\lim _{\varepsilon \rightarrow 0} \int_{D \backslash \bar{E}} \phi\left(x, \frac{x}{\varepsilon}, m_{\varepsilon}\right)+\frac{1}{2}\left|\nabla u_{\varepsilon}\right| \mathrm{d} x \leq \mathcal{F}^{-}(m ; D \backslash \bar{E})+\eta \\
\lim _{\varepsilon \rightarrow 0} \int_{B} \phi\left(x, \frac{x}{\varepsilon}, w_{\varepsilon}\right)+\frac{1}{2}\left|\nabla z_{\varepsilon}\right| \mathrm{d} x \leq \mathcal{F}^{-}(m ; B)+\eta .
\end{gathered}
$$

Since by Lemma $2.2\left\{\nabla u_{\varepsilon}\right\}$ and $\left\{\nabla z_{\varepsilon}\right\}$ are 2-equiintegrable, we have that there exists $K>0$ such that

$$
\int_{B \backslash \bar{E}}\left(\left|\nabla u_{\varepsilon}\right|^{2}+\left|\nabla z_{\varepsilon}\right|^{2}\right) \mathrm{d} x \leq K
$$

and we can find an open set $S$ with $E \subset \subset S \subset \subset B$ such that for $j$ sufficiently large we have

$$
\int_{S_{j}}\left(a(x)+\left|\nabla u_{\varepsilon}\right|^{2}+\left|\nabla z_{\varepsilon}\right|^{2}\right) \mathrm{d} x<\varepsilon
$$

where

$$
S_{j}:=\left\{x \in S: \frac{1}{j+1} \leq \operatorname{dist}(x, \partial S) \leq \frac{1}{j}\right\}
$$

Let $\theta_{j}$ be cut-off functions with compact support in $S$, which are equal to 1 when $\operatorname{dist}(x, \partial S) \geq \frac{1}{j}$ and 0 when $\operatorname{dist}(x, \partial S) \leq \frac{1}{j+1}$. For $x \in D$ setting

$$
\begin{gathered}
\widehat{M}_{j, \varepsilon}:=\left(1-\theta_{j}\right) m_{\varepsilon}+\theta_{j} w_{\varepsilon} \\
M_{j, \varepsilon}:=\pi\left(\widehat{M}_{j, \varepsilon}\right) \quad \text { with } \pi(m):= \begin{cases}\frac{m}{|m|} \text { if } m \neq 0 \\
e_{1} \text { if } m=0\end{cases} \\
\widehat{U}_{j, \varepsilon}:=\left(1-\theta_{j}\right) u_{\varepsilon}+\theta_{j} z_{\varepsilon},
\end{gathered}
$$

we have (See [13], Lem. 6) that $\widehat{M}_{j, \varepsilon} \stackrel{*}{\rightarrow} m$ in $L^{\infty}\left(D ; \mathbb{R}^{3}\right)$ for $\varepsilon \rightarrow 0$ and $j \rightarrow 0$ and for $j$ fixed

$$
\Delta \widehat{U}_{j, \varepsilon}+\operatorname{div} \widehat{M}_{j, \varepsilon} \rightarrow 0 \quad \text { in } H^{-1}(D)
$$

Now we can say

$$
\begin{aligned}
\int_{D} \phi\left(x, \frac{x}{\varepsilon}, M_{j, \varepsilon}\right)+\frac{1}{2}\left|\nabla \widehat{U}_{j, \varepsilon}\right|^{2} \mathrm{~d} x \leq & \int_{D \backslash E} \phi\left(x, \frac{x}{\varepsilon}, m_{\varepsilon}\right)+\frac{1}{2}\left|\nabla u_{\varepsilon}\right|^{2} \mathrm{~d} x \\
& +\int_{B} \phi\left(x, \frac{x}{\varepsilon}, w_{\varepsilon}\right)+\frac{1}{2}\left|\nabla z_{\varepsilon}\right|^{2} \mathrm{~d} x+\int_{S_{j}} \phi\left(x, \frac{x}{\varepsilon}, M_{j, \varepsilon}\right)+\frac{1}{2}\left|\nabla \widehat{U}_{j, \varepsilon}\right|^{2} \mathrm{~d} x \\
\leq & \int_{D \backslash \bar{E}} \cdots \mathrm{d} x+\int_{B} \cdots \mathrm{d} x+\int_{S_{j}} a(x) \mathrm{d} x+\int_{S_{j}} \frac{1}{2}\left|\nabla u_{\varepsilon}\right|^{2}+\frac{1}{2}\left|\nabla z_{\varepsilon}\right|^{2} \mathrm{~d} x \\
& +C \int_{S_{j}}\left|\nabla \theta_{j}\right|^{2}\left|u_{\varepsilon}-z_{\varepsilon}\right|^{2} \mathrm{~d} x
\end{aligned}
$$

and since $u_{\varepsilon}-z_{\varepsilon} \rightarrow 0$ in $H^{1}(B \backslash \bar{E}), S_{j} \subset B \backslash \bar{E}$ for large $j$ and $\left|S_{j}\right| \rightarrow 0$, using (3.5), letting $\varepsilon \rightarrow 0$ and $j \rightarrow \infty$, we obtain

$$
\liminf _{j \rightarrow \infty} \liminf _{\varepsilon \rightarrow 0} \int_{D} \phi\left(x, \frac{x}{\varepsilon}, M_{j, \varepsilon}\right)+\frac{1}{2}\left|\nabla \widehat{U}_{j, \varepsilon}\right|^{2} \mathrm{~d} x \leq \mathcal{F}^{-}(m ; D \backslash \bar{E})+\mathcal{F}^{-}(m ; B)+3 \eta
$$


Let $U_{j, \varepsilon} \in u+H_{0}^{1}(D)$ be the solution of $\Delta U_{j, \varepsilon}+\operatorname{div} M_{j, \varepsilon}=0$ in $D$. Observe that, by definition of $\widehat{M}_{j, \varepsilon}$ and $M_{j, \varepsilon}$ we have for any fixed $p \geq 1$

$$
\int_{D}\left|\widehat{M}_{j, \varepsilon}-M_{j, \varepsilon}\right|^{p} d x \leq 2^{p}\left|S_{j}\right| \rightarrow 0 \text { as } j \rightarrow \infty
$$

hence, by (3.6)

$$
\lim _{j \rightarrow \infty} \lim _{\varepsilon \rightarrow 0} \Delta\left(\widehat{U}_{j, \varepsilon}-U_{j, \varepsilon}\right)=\lim _{j \rightarrow \infty} \lim _{\varepsilon \rightarrow 0} \operatorname{div}\left(-\widehat{M}_{j, \varepsilon}+M_{j, \varepsilon}\right)=0 \quad \text { in } H^{-1}(D) .
$$

So since $\widehat{U}_{j, \varepsilon}-U_{j, \varepsilon} \in H_{0}^{1}(D)$, by definition we have

$$
\lim _{j \rightarrow \infty} \lim _{\varepsilon \rightarrow 0}\left\|\nabla\left(\widehat{U}_{j, \varepsilon}-U_{j, \varepsilon}\right)\right\|_{L^{2}\left(D ; \mathbb{R}^{3}\right)}=0 .
$$

Now we are in the position to say that

$$
\begin{aligned}
\liminf _{j \rightarrow \infty} \liminf _{\varepsilon \rightarrow 0} \int_{D} \phi\left(x, \frac{x}{\varepsilon}, M_{j, \varepsilon}\right)+\frac{1}{2}\left|\nabla U_{j, \varepsilon}\right|^{2} \mathrm{~d} x & \leq \liminf _{j \rightarrow \infty} \liminf _{\varepsilon \rightarrow 0} \int_{D} \phi\left(x, \frac{x}{\varepsilon}, M_{j, \varepsilon}\right)+\frac{1}{2}\left|\nabla \widehat{U}_{j, \varepsilon}\right|^{2} \mathrm{~d} x \\
& +\liminf _{j \rightarrow \infty} \liminf _{\varepsilon \rightarrow 0} \int_{D} \frac{1}{2}\left|\nabla \widehat{U}_{j, \varepsilon}-\nabla U_{j, \varepsilon}\right|^{2} \mathrm{~d} x \\
& =\liminf _{j \rightarrow \infty} \liminf _{\varepsilon \rightarrow 0} \int_{D} \phi\left(x, \frac{x}{\varepsilon}, M_{j, \varepsilon}\right)+\frac{1}{2}\left|\nabla \widehat{U}_{j, \varepsilon}\right|^{2} \mathrm{~d} x \\
& \leq \mathcal{F}^{-}(m ; D \backslash \bar{E})+\mathcal{F}^{-}(m ; B)+3 \eta .
\end{aligned}
$$

Finally using a diagonalization argument and letting $\eta \rightarrow 0$ we found $U_{\varepsilon}$ and $M_{\varepsilon} \stackrel{*}{\rightarrow} m$ such that $\Delta U_{\varepsilon}+\operatorname{div} M_{\varepsilon}=$ 0 in $D$ and

$$
\liminf _{\varepsilon \rightarrow 0} \int_{D} \phi\left(x, \frac{x}{\varepsilon}, M_{\varepsilon}\right)+\frac{1}{2}\left|\nabla U_{\varepsilon}\right|^{2} \mathrm{~d} x \leq \mathcal{F}^{-}(m ; D \backslash \bar{E})+\mathcal{F}^{-}(m ; B),
$$

and taking the infimum we have

$$
\mathcal{F}^{-}(m ; D) \leq \mathcal{F}^{-}(m ; D \backslash \bar{E})+\mathcal{F}^{-}(m ; B) .
$$

In particular $\mathcal{F}^{-}(m ; \cdot)$ as a set function is absolutely continuous with respect to Lebesgue measure on $\mathbb{R}^{3}$, and we are interested in finding its Radon-Nikodỳm derivative in order to obtain an explicit formula for the density function in the integral representation of $\mathcal{F}^{-}$.

Suppose now that the anisotropy energy, $\phi$, does not depend on the position, that is $\phi:\left(\mathbb{R}^{3} \times S^{2} \cup\{0\}\right) \rightarrow$ $[0, \infty)$ and define for $(\mu, h) \in \bar{B} \times \mathbb{R}^{3}$

$$
f_{\text {hom }}(\mu, h):=\inf _{S_{\mu}}\left\{\liminf _{k \rightarrow \infty} \int_{Q} \phi\left(k y, M_{k}(y)\right)+\frac{1}{2}\left|h+\nabla U_{k}(y)\right|^{2} \mathrm{~d} y\right\}
$$

where $Q$ is the unit cube in $\mathbb{R}^{3}$ and

$$
S_{\mu}:=\left\{\begin{array}{c}
\left\{M_{k}, U_{k}\right\} \in L^{\infty}\left(Q, \mathbb{R}^{3}\right) \times\left(H_{0}^{1}(Q)\right):\left|M_{k}\right|=\chi_{Q}, \\
M_{k} \stackrel{*}{\rightarrow} \operatorname{in} L^{\infty}\left(Q, \mathbb{R}^{3}\right), \operatorname{div} M_{k}+\Delta U_{k}=0 \text { in } Q
\end{array}\right\}
$$

Remark 3.6. We want to point out that the we can prove the upper semicontinuity of $f_{\text {hom }}$ using the same argument used in [13] (see Lems. 8 and 9 in [13]). 
Now we are in position to state the following

Proposition 3.7. For every $m \in \mathcal{M}^{*}$ and for a.e. $x_{0} \in \Omega$ we have

$$
\frac{\mathrm{d} \mathcal{F}^{-}(m ; \cdot)}{\mathrm{d} \mathcal{L}^{3}}\left(x_{0}\right)=f_{\text {hom }}\left(m\left(x_{0}\right), \nabla u\left(x_{0}\right)\right),
$$

where $u \in H^{1}\left(\mathbb{R}^{3}\right)$ is the unique solution of $\Delta u+\operatorname{div} m=0$ in $\mathbb{R}^{3}$.

Proof. In order to simplify the notations we will represent the subsequence $\left\{\varepsilon_{n_{k}}\right\}$ by $\left\{\varepsilon_{n}\right\}$ or simply by $\{\varepsilon\}$. We divide the proof in four steps.

Step 1 (translation invariance). We claim that

$$
\mathcal{F}^{-}(m ; D)=\mathcal{F}^{-}\left(m\left(\cdot-x_{0}\right) ; D+x_{0}\right)
$$

Proof of Step 1. Let $\left\{m_{\varepsilon}, u_{\varepsilon}\right\} \in \mathbf{M}_{\mathbf{D}}^{\prime}$ such that

$$
\mathcal{F}^{-}(m ; D)=\liminf _{\varepsilon \rightarrow 0} \mathcal{F}_{\varepsilon}\left(m_{\varepsilon} ; D\right)=\liminf _{\varepsilon \rightarrow 0} \int_{D} \phi\left(\frac{x}{\varepsilon}, m_{\varepsilon}\right)+\frac{1}{2}\left|\nabla u_{\varepsilon}\right|^{2} \mathrm{~d} x
$$

Consider the sequence $z_{\varepsilon}=\left[\frac{x_{0}}{\varepsilon}\right] \in \mathbb{Z}^{3}$, we have $x_{\varepsilon}:=z_{\varepsilon} \varepsilon \rightarrow x_{0}$ and by periodicity of $\phi$

$$
\begin{aligned}
\mathcal{F}_{\varepsilon}\left(m_{\varepsilon} ; D\right) & =\int_{D} \phi\left(\frac{x+x_{\varepsilon}}{\varepsilon}, m_{\varepsilon}(x)\right)+\frac{1}{2}\left|\nabla u_{\varepsilon}(x)\right|^{2} \mathrm{~d} x \\
& =\int_{D+x_{\varepsilon}} \phi\left(\frac{y}{\varepsilon}, m_{\varepsilon}\left(y-x_{\varepsilon}\right)\right)+\frac{1}{2}\left|\nabla u_{\varepsilon}\left(y-x_{\varepsilon}\right)\right|^{2} \mathrm{~d} y .
\end{aligned}
$$

Let $B \subset \subset D$, for $\varepsilon$ small enough we have that $D+x_{\varepsilon} \supset B+x_{0}$ and thus

$$
\mathcal{F}_{\varepsilon}\left(m_{\varepsilon} ; D\right) \geq \int_{B+x_{0}} \phi\left(\frac{y}{\varepsilon}, m_{\varepsilon}\left(y-x_{\varepsilon}\right)\right)+\frac{1}{2}\left|\nabla u_{\varepsilon}\left(y-x_{\varepsilon}\right)\right|^{2} \mathrm{~d} y .
$$

Since $m_{\varepsilon}\left(\cdot-x_{\varepsilon}\right) \stackrel{*}{\rightarrow} m\left(\cdot-x_{0}\right)$ in $L^{\infty}\left(B+x_{0}, \mathbb{R}^{3}\right), \operatorname{div}\left(m_{\varepsilon}\left(\cdot-x_{\varepsilon}\right)+\nabla u_{\varepsilon}\left(\cdot-x_{\varepsilon}\right)\right)=0$ in $B+x_{0}$ and $\nabla u_{\varepsilon}\left(\cdot-x_{\varepsilon}\right) \rightarrow$ $\nabla u\left(\cdot-x_{0}\right)$ in $L^{2}\left(B+x_{0} ; \mathbb{R}^{3}\right)$, by $(3.7)$ we obtain

$$
\mathcal{F}^{-}(m ; D) \geq \mathcal{F}^{-}\left(m\left(\cdot-x_{0}\right) ; B+x_{0}\right)
$$

By setting $B_{m}:=R_{m}-x_{0}$, where $R_{m} \in \mathcal{R}(\Omega)$ and $R_{m} \nearrow D+x_{0}$, we obtain, using the inner regularity that

$$
\mathcal{F}^{-}(m ; D) \geq \mathcal{F}^{-}\left(m\left(\cdot-x_{0}\right) ; D+x_{0}\right)
$$

The converse inequality can be proved following the same argument.

Step 2 (lower bound). We have that

$$
\frac{\mathrm{d} \mathcal{F}^{-}(m ; \cdot)}{\mathrm{d} \mathcal{L}^{3}}\left(x_{0}\right) \geq f_{\text {hom }}\left(m\left(x_{0}\right), \nabla u\left(x_{0}\right)\right) .
$$

Proof of Step 2. Let $\left\{m_{\varepsilon}, u_{\varepsilon}\right\} \in \mathbf{M}_{Q\left(x_{0}, r\right)}^{\prime}$, where $Q\left(x_{0}, r\right)$ is the cube centered in $x_{0}$ and with side $r$, such that

$$
\lim _{\varepsilon \rightarrow 0} \int_{Q\left(x_{0}, r\right)} \phi\left(\frac{x}{\varepsilon}, m_{\varepsilon}(x)\right)+\frac{1}{2}\left|\nabla u_{\varepsilon}(x)\right|^{2} \mathrm{~d} x \leq \mathcal{F}^{-}\left(m, Q\left(x_{0}, r\right)\right)+r^{4}
$$


We have $m_{\varepsilon}\left(\cdot+x_{0}\right) \stackrel{*}{\rightarrow} m\left(\cdot+x_{0}\right)$ in $L^{\infty}\left(Q(0, r), \mathbb{R}^{3}\right)$ and $\Delta u_{\varepsilon}\left(\cdot+x_{0}\right)+\operatorname{div} m_{\varepsilon}\left(\cdot+x_{0}\right)=0$ in $Q(0, r)$. So we can say, by definition of Radon-Nikodým derivative and by Step 1, that

$$
\begin{aligned}
\frac{d \mathcal{F}^{-}(m ; \cdot)}{d \mathcal{L}^{3}}\left(x_{0}\right) & =\lim _{r \rightarrow 0} \frac{\mathcal{F}^{-}\left(m ; Q\left(x_{0}, r\right)\right)}{r^{3}}=\lim _{r \rightarrow 0} \frac{\mathcal{F}^{-}\left(m\left(\cdot+x_{0}\right) ; Q(0, r)\right)}{r^{3}} \\
& \geq \limsup _{r \rightarrow 0} \lim _{\varepsilon \rightarrow 0} f_{r Q} \phi\left(\frac{x}{\varepsilon}, m_{\varepsilon}\left(x+x_{0}\right)\right)+\frac{1}{2}\left|\nabla u_{\varepsilon}\left(x+x_{0}\right)\right|^{2} \mathrm{~d} x \\
& =\limsup _{r \rightarrow 0} \lim _{\varepsilon \rightarrow 0} \int_{Q} \phi\left(\frac{r}{\varepsilon} y, m_{r, \varepsilon}(y)\right)+\frac{1}{2}\left|\nabla u_{r, \varepsilon}(y)\right|^{2} \mathrm{~d} y
\end{aligned}
$$

where

$$
m_{r, \varepsilon}:=m_{\varepsilon}\left(r y+x_{0}\right) \quad \text { and } \quad u_{r, \varepsilon}:=\frac{u_{\varepsilon}\left(r y+x_{0}\right)+u_{\varepsilon}\left(x_{0}\right)}{r} .
$$

Moreover we have (see [13], Lem. 7 for a proof) that:

(1) $m_{r, \varepsilon} \stackrel{*}{\rightarrow} m\left(x_{0}\right)$ in $L^{\infty}\left(Q, \mathbb{R}^{3}\right)$ if first $\varepsilon \rightarrow 0$ then $r \rightarrow 0$;

(2) $u_{r, \varepsilon} \rightarrow u_{0}(y)=\nabla u\left(x_{0}\right) y$ in $H^{1}(Q)$;

(3) the sequence $\left\{m_{r, \varepsilon}, u_{r, \varepsilon}\right\} \in L^{\infty}\left(Q, S^{2}\right) \times\left(u_{r}+H_{0}^{1}(Q)\right)$ is such that

$$
\Delta u_{r, \varepsilon}+\operatorname{div} m_{r, \varepsilon}=0 \text { in } Q
$$

where

$$
u_{r}(y)=\frac{u\left(x_{0}+r y\right)-u\left(x_{0}\right)}{r}
$$

Diagonalize to get the subsequences $\hat{m}_{k}=m_{r_{k}, \varepsilon_{k}}$ and $\hat{u}_{k}=u_{r_{k}, \varepsilon_{k}}$ with

$$
\begin{gathered}
\left\{\left(\hat{m}_{k}, \hat{u}_{k}\right)\right\} \in L^{\infty}\left(Q, S^{2}\right) \times\left(u_{r_{k}}+H_{0}^{1}(Q)\right), \\
\Delta \hat{u}_{k}+\operatorname{div} \hat{m}_{k}=0 \quad \text { in } Q
\end{gathered}
$$

such that

$$
\begin{aligned}
& \hat{m}_{k} \stackrel{*}{\rightarrow} m\left(x_{0}\right) \text { in } L^{\infty}\left(Q, \mathbb{R}^{3}\right), \hat{u}_{k} \rightarrow u_{0}(y) \text { in } H^{1}(Q) \\
& \frac{\mathrm{d} \mathcal{F}^{-}(m ; \cdot)}{\mathrm{d} \mathcal{L}^{3}}\left(x_{0}\right) \geq \lim _{k \rightarrow \infty} \int_{Q} \phi\left(s_{k} y, \hat{m}_{k}(y)\right)+\frac{1}{2}\left|\nabla \hat{u}_{k}\right|^{2} \mathrm{~d} y
\end{aligned}
$$

with $s_{k}=\frac{r_{k}}{\varepsilon_{k}} \rightarrow \infty$.

Set $\hat{v}_{k}(y)=\hat{u}_{k}(y)-u_{0}(y)$, we have

$$
\frac{\mathrm{d} \mathcal{F}^{-}(m ; \cdot)}{\mathrm{d} \mathcal{L}^{3}}\left(x_{0}\right) \geq \lim _{k \rightarrow \infty} \int_{Q} \phi\left(s_{k} y, \hat{m}_{k}(y)\right)+\frac{1}{2}\left|\nabla u_{0}\left(x_{0}\right)+\nabla \hat{v}_{k}(y)\right|^{2} \mathrm{~d} y .
$$

Let $v_{k} \in H_{0}^{1}(Q)$ be the unique solution of $\Delta v_{k}+\operatorname{div} \hat{m}_{k}=0$ in $Q$, since

$$
\left\{\begin{array}{l}
\Delta\left(\hat{v}_{k}-v_{k}\right)=0 \quad \text { on } Q \\
\hat{v}_{k}-v_{k}=\hat{u}_{k}-u_{0} \text { in } \partial Q
\end{array}\right.
$$

as $\hat{u}_{k}-u_{0} \rightarrow 0$ in $H^{1}(Q)$ and $\left\{\nabla \hat{u}_{k}\right\}$ is 2-equi-integrable over $Q$, by Lemma 2.2 it follows that $\left\|\hat{v}_{k}-v_{k}\right\|_{H^{1}(Q)} \rightarrow 0$ and so

$$
\lim _{k \rightarrow \infty} \int_{Q}\left|\nabla u_{0}\left(x_{0}\right)+\nabla \hat{v}_{k}(y)\right|^{2} \mathrm{~d} y=\lim _{k \rightarrow \infty} \int_{Q}\left|\nabla u_{0}\left(x_{0}\right)+\nabla v_{k}(y)\right|^{2} \mathrm{~d} y .
$$


Using (3.8), (3.9) we can write

$$
\begin{aligned}
\frac{\mathrm{d} \mathcal{F}^{-}(m ; \cdot)}{\mathrm{d} \mathcal{L}^{3}}\left(x_{0}\right) & \geq \lim _{k \rightarrow \infty} \int_{Q} \phi\left(s_{k} y, \hat{m}_{k}(y)\right)+\frac{1}{2}\left|\nabla u_{0}\left(x_{0}\right)+\nabla v_{k}(y)\right|^{2} \mathrm{~d} y \\
& \geq \liminf _{k \rightarrow \infty} \frac{1}{s_{k}^{3}} \int_{\left(\left[s_{k}\right]+1\right) Q} \phi\left(x, \hat{m}_{k}\left(\frac{x}{s_{k}}\right)\right)+\frac{1}{2}\left|\nabla u_{0}\left(x_{0}\right)+\nabla v_{k}\left(\frac{x}{s_{k}}\right)\right|^{2} \mathrm{~d} x \\
& -\underbrace{\limsup _{k \rightarrow \infty} \frac{1}{s_{k}^{3}} \int_{\left(\left[s_{k}\right]+1\right) Q \backslash s_{k} Q} \phi\left(x, \hat{m}_{k}\left(\frac{x}{s_{k}}\right)\right)+\frac{1}{2}\left|\nabla u_{0}\left(x_{0}\right)+\nabla v_{k}\left(\frac{x}{s_{k}}\right)\right|^{2} \mathrm{~d} x}_{A} .
\end{aligned}
$$

We claim that $A=0$, indeed we can write

$$
A=\limsup _{k \rightarrow \infty} \int_{\frac{\left(\left[s_{k}\right]+1\right)}{s_{k}} Q \backslash Q} \phi\left(s_{k} x, \hat{m}_{k}(y)\right)+\frac{1}{2}\left|\nabla u_{0}\left(x_{0}\right)+\nabla v_{k}(y)\right|^{2} \mathrm{~d} x
$$

and since

$$
\frac{\left[s_{k}\right]+1}{s_{k}} \rightarrow 1 \Rightarrow\left|\frac{\left(\left[s_{k}\right]+1\right)}{s_{k}} Q \backslash Q\right|=\frac{\left[s_{k}\right]+1}{s_{k}}-1 \rightarrow 0,
$$

the 2-equi-integrability of $\left\{\nabla v_{k}\right\}$ and the growth condition (1.4) imply $A=0$.

Setting now $h_{k}=\frac{1}{s_{k}} ; n_{k}=\left[s_{k}\right]+1 \in \mathbb{N}$, we have $1<h_{k} n_{k} \rightarrow 1$ and

$$
\begin{aligned}
\frac{\mathrm{d} \mathcal{F}^{-}(m ; \cdot)}{\mathrm{d} \mathcal{L}^{3}}\left(x_{0}\right) & \geq \liminf _{k \rightarrow \infty} \frac{\left(h_{k} n_{k}\right)^{3}}{n_{k}^{3}} \int_{n_{k} Q} \phi\left(x, \hat{m}_{k}\left(h_{k} x\right)\right)+\frac{1}{2}\left|\nabla u_{0}\left(x_{0}\right)+\nabla v_{k}\left(h_{k} x\right)\right|^{2} \mathrm{~d} x \\
& =\liminf _{k \rightarrow \infty} \int_{Q} \phi\left(n_{k} x, \hat{m}_{k}\left(n_{k} h_{k} x\right)\right)+\left|\nabla u_{0}\left(x_{0}\right)+\frac{1}{2} \nabla v_{k}\left(n_{k} h_{k} x\right)\right|^{2} \mathrm{~d} x .
\end{aligned}
$$

Set $\tilde{m}_{k}(y)=\hat{m}_{k}\left(n_{k} h_{k} y\right), \quad \tilde{v}_{k}(y)=v_{k}\left(n_{k} h_{k} y\right)$, we have

$$
\tilde{m}_{k}(y) \stackrel{*}{\rightarrow} m\left(x_{0}\right) \text { in } L^{\infty}\left(Q, \mathbb{R}^{3}\right) \text { and } \tilde{v}_{k}(y) \rightarrow 0 \text { in } H^{1}(Q) .
$$

Note that the functions $\hat{m}_{k}$ and $v_{k}$ are supposed extended by periodicity.

Now let $\bar{v}_{k} \in H_{0}^{1}(Q)$ be the unique solution of $\Delta \bar{v}_{k}+\operatorname{div} \tilde{m}_{k}=0$ in $Q$, we claim that $\left\|\bar{v}_{k}-\tilde{v}_{k}\right\|_{H^{1}(Q)} \rightarrow 0$. Indeed, since $Q \subset n_{k} h_{k} Q$ and by definition of $\tilde{v}_{k}$, we have

$$
\left\{\begin{array}{l}
\Delta\left(\tilde{v}_{k}-\bar{v}_{k}\right)=0 \text { in } Q \\
\tilde{v}_{k}-\bar{v}_{k}=\tilde{v}_{k} \quad \text { on } \partial Q
\end{array}\right.
$$

by Lemma 2.2 with $\phi_{k}=\tilde{v}_{k}$ and $m_{k}=0$ we have

$$
\tilde{v}_{k}-\bar{v}_{k} \rightarrow 0 \text { in } H^{1}(Q)
$$

Finally we have

$$
\begin{aligned}
\frac{\mathrm{d} \mathcal{F}^{-}(m ; \cdot)}{\mathrm{d} \mathcal{L}^{3}}\left(x_{0}\right) & \geq \liminf _{k \rightarrow \infty} \int_{Q} \phi\left(n_{k} x, \tilde{m}_{k}(x)\right)+\frac{1}{2}\left|\nabla u_{0}\left(x_{0}\right)+\nabla \bar{v}_{k}(x)\right|^{2} \mathrm{~d} x \\
& \geq f_{\text {hom }}\left(m\left(x_{0}\right), \nabla u\left(x_{0}\right)\right) .
\end{aligned}
$$


Step 3 (upper bound for piecewise constant magnetizations). We prove that if $m \in \mathcal{M}^{*}$ is such that

$$
m(x)=\sum_{i=1}^{k} \xi_{i} \chi_{D_{i}}(x)
$$

where $\left|\xi_{i}\right| \leq 1$ and the sets $D_{i}$ are pairwise disjoint, $\left|\partial D_{i}\right|=0$ and $\bigcup_{i=0}^{k} \overline{D_{i}}=\Omega$, then:

$$
\frac{\mathrm{d} \mathcal{F}^{-}(m ; \cdot)}{\mathrm{d} \mathcal{L}^{3}}\left(x_{0}\right) \leq f_{\text {hom }}\left(m\left(x_{0}\right), \nabla u\left(x_{0}\right)\right) .
$$

Proof of Step 3. Assume that $x_{0} \in \bigcup_{i=0}^{k} D_{i}$, say $x_{0} \in D_{1}$, so $m(x)=m\left(x_{0}\right)=\xi_{1} \forall x \in Q\left(x_{0}, r\right)$ for $r$ small enough.

Without loss of generality we may assume also that

$$
\frac{u\left(x_{0}+r y\right)-u\left(x_{0}\right)}{r}-\nabla u\left(x_{0}\right) y \rightarrow 0 \text { in } H^{1}(Q)
$$

Note that (3.11) means that $x_{0}$ is a 2-Lebesgue point for $\nabla u$ and a point of 2-approximate differentiability for $u$.

For all $\eta$ fixed we can find, by definition of $f_{\text {hom }},\left\{\left(M_{k}, U_{k}\right)\right\} \in S_{m\left(x_{0}\right)}$ such that

$$
\lim _{n \rightarrow \infty} \int_{Q} \phi\left(k y, M_{k}(y)\right)+\frac{1}{2}\left|\nabla u\left(x_{0}\right)+\nabla U_{k}(y)\right|^{2} \mathrm{~d} y \leq f_{\text {hom }}\left(m\left(x_{0}\right), \nabla u\left(x_{0}\right)\right)+\eta .
$$

Set $\gamma_{n, r}=\frac{r}{\varepsilon_{n}}\left[\frac{r}{\varepsilon_{n}}\right]^{-1} \geq 1$ and $k_{n}=\left[\frac{r}{\varepsilon_{n}}\right]$, where we have used the notation $\left\{\varepsilon_{n}\right\}$ for $\left\{\varepsilon_{n_{k}}\right\}$. For any fixed $r<<1$ define

$$
\widetilde{M}_{n}(x)=M_{k_{n}}\left(\frac{\left(x-x_{0}\right) \gamma_{n, r}}{r}\right)
$$

and remark that for $r$ small enough we have $m(x)=m\left(x_{0}\right)$ in $Q\left(x_{0}, r\right)$. We claim that $\widetilde{M}_{n} \stackrel{*}{\rightarrow} m(x)$ in $L^{\infty}\left(Q\left(x_{0}, r\right)\right)$. Indeed to prove the $L^{\infty}$ weak-* convergence of $\widetilde{M}_{n}$ we only have to show (see [6], Lem. 1.4) that

$$
\lim _{n \rightarrow \infty} \int_{D}\left(\widetilde{M}_{n}-m\left(x_{0}\right)\right) \mathrm{d} x=0 \text { for all cube } D \subset Q\left(x_{0}, r\right),
$$

since we already know that $\left\|\widetilde{M}_{n}\right\|_{\infty} \leq 1$.

Let $D \subset Q\left(x_{0}, r\right)$ a cube, we have, using a change of variables

$$
\int_{D}\left(\widetilde{M}_{n}-m\left(x_{0}\right)\right) \mathrm{d} x=\frac{1}{\gamma_{n, r}^{3}} \int_{\gamma_{n, r}\left(D-x_{0}\right)}\left(M_{k_{n}}\left(\frac{y}{r}\right)-m\left(x_{0}\right)\right) \mathrm{d} y=A_{n}+B_{n}
$$

where

Now observe that

$$
\begin{gathered}
A_{n}=\frac{1}{\gamma_{n, r}^{3}} \int_{\gamma_{n, r}\left(D-x_{0}\right) \backslash\left(D-x_{0}\right)}\left(M_{k_{n}}\left(\frac{y}{r}\right)-m\left(x_{0}\right)\right) \mathrm{d} y \\
B_{n}=\frac{1}{\gamma_{n, r}^{3}} \int_{\left(D-x_{0}\right)}\left(M_{k_{n}}\left(\frac{y}{r}\right)-m\left(x_{0}\right)\right) \mathrm{d} y .
\end{gathered}
$$

$$
\begin{aligned}
\limsup _{n \rightarrow \infty}\left|A_{n}\right| & \leq \limsup _{n \rightarrow \infty} \int_{\gamma_{n, r}\left(D-x_{0}\right) \backslash\left(D-x_{0}\right)}\left|M_{k_{n}}\left(\frac{y}{r}\right)-m\left(x_{0}\right)\right| \mathrm{d} y \\
& \leq 2 \lim _{n \rightarrow \infty}\left|\gamma_{n, r}\left(D-x_{0}\right) \backslash\left(D-x_{0}\right)\right|=0
\end{aligned}
$$


and that

$$
\limsup _{n \rightarrow \infty}\left|B_{n}\right|=r^{3}\left|\int_{\frac{D-x_{0}}{r}}\left(M_{k_{n}}(x)-m\left(x_{0}\right)\right)\right| \mathrm{d} x=0
$$

since $M_{k_{n}} \stackrel{*}{\rightarrow} m\left(x_{0}\right)$ in $L^{\infty}(Q)$ and the cube $\frac{D-x_{0}}{r} \subset Q$. So we have (3.12) since

$$
\lim _{n \rightarrow \infty}\left|\int_{D}\left(\widetilde{M}_{n}-m\left(x_{0}\right)\right) \mathrm{d} x\right| \leq \limsup _{n \rightarrow \infty}\left|A_{n}\right|+\limsup _{n \rightarrow \infty}\left|B_{n}\right| .
$$

Using the sequence $\widetilde{M}_{n}$ in the definition of $\mathcal{F}$, setting $\widetilde{U}_{n} \in u+H_{0}^{1}\left(Q\left(x_{0}, r\right)\right)$ the unique solution of $\Delta \widetilde{U}_{n}+$ $\operatorname{div} \widetilde{M}_{n}=0$ in $Q\left(x_{0}, r\right)$ and using Step 1, we can write

$$
\begin{aligned}
\frac{\mathrm{d} \mathcal{F}(m ; \cdot)}{\mathrm{d} \mathcal{L}^{3}}\left(x_{0}\right)= & \lim _{r \rightarrow 0} \frac{\mathcal{F}\left(m\left(\cdot+x_{0}\right) ; r Q\right)}{r^{3}} \\
\leq & \liminf _{r \rightarrow 0} \liminf _{n \rightarrow \infty} \frac{1}{r^{3}} \int_{r Q} \phi\left(\frac{x}{\varepsilon_{n}}, \widetilde{M}_{n}\left(x+x_{0}\right)\right)+\frac{1}{2}\left|\nabla \widetilde{U}_{n}\left(x+x_{0}\right)\right|^{2} \mathrm{~d} x \\
= & \liminf _{r \rightarrow 0} \liminf _{n \rightarrow \infty} \frac{1}{r^{3} \gamma_{n, r}^{3}} \int_{\gamma_{n, r} r Q} \phi\left(\left[\frac{r}{\varepsilon_{n}}\right] \frac{y}{r}, \widetilde{M}_{n}\left(\frac{y}{\gamma_{n, r}}+x_{0}\right)\right) \\
& +\frac{1}{2}\left|\nabla \widetilde{U}_{n}\left(\frac{y}{\gamma_{n, r}}+x_{0}\right)\right|^{2} \mathrm{~d} y \\
= & B+C,
\end{aligned}
$$

where

$$
B=\liminf _{r \rightarrow 0} \liminf _{n \rightarrow \infty} \frac{1}{r^{3}} \int_{r Q} \phi\left(\left[\frac{r}{\varepsilon_{n}}\right] \frac{y}{r}, M_{k_{n}}\left(\frac{y}{r}\right)\right)+\frac{1}{2}\left|\nabla \widetilde{U}_{n}\left(\frac{y}{\gamma_{n, r}}+x_{0}\right)\right|^{2} \mathrm{~d} y
$$

and

$$
C=\liminf _{r \rightarrow 0} \liminf _{n \rightarrow \infty} \frac{1}{r^{3}} \int_{\gamma_{n, r} r Q \backslash r Q} \phi\left(\left[\frac{r}{\varepsilon_{n}}\right] \frac{y}{r}, M_{k_{n}}\left(\frac{y}{r}\right)\right)+\frac{1}{2}\left|\nabla \widetilde{U}_{n}\left(\frac{y}{\gamma_{n, r}}+x_{0}\right)\right|^{2} \mathrm{~d} y .
$$

Using the same argument as in Step 2 we can show that $C=0$ since $\left|\gamma_{n, r} r Q \backslash r Q\right|$ goes to zero as $n \rightarrow \infty$.

Moreover setting

$$
\begin{aligned}
& V_{k_{n}}(y)=y \nabla u\left(x_{0}\right)+U_{k_{n}}(y) \\
& \bar{U}_{n}(y)=\frac{\widetilde{U}_{n}\left(\frac{x r}{\gamma_{n, r}}+x_{0}\right)-u\left(x_{0}\right)}{\frac{r}{\gamma_{n, r}}} .
\end{aligned}
$$

Since

$$
\left\{\begin{array}{l}
\Delta\left(V_{k_{n}}(y)-\bar{U}_{n}(y)\right)=0 \\
V_{k_{n}}(y)-\bar{U}_{n}(y)=y \nabla u\left(x_{0}\right)-\frac{u\left(\frac{x r}{\gamma_{n, r}}+x_{0}\right)-u\left(x_{0}\right)}{\frac{r}{\gamma_{n, r}}} \text { in } Q
\end{array}\right.
$$

by Lemma 2.2 and by (3.11) we have $\left\|V_{k_{n}}-\bar{U}_{n}\right\|_{H^{1}(Q)} \rightarrow 0$ as $r \rightarrow 0$.

So we can say, changing variables, using the definition of $k_{n}$ and the equality

$$
\nabla \bar{U}_{n}(y)=\nabla \widetilde{U}_{n}\left(\frac{x r}{\gamma_{n, r}}+x_{0}\right)
$$


that

$$
\begin{aligned}
B & =\liminf _{r \rightarrow 0} \liminf _{n \rightarrow \infty} \int_{Q} \phi\left(\left[\frac{r}{\varepsilon_{n}}\right] y, M_{k_{n}}(y)\right)+\frac{1}{2}\left|\nabla \widetilde{U}_{n}\left(\frac{y r}{\gamma_{n, r}}+x_{0}\right)\right|^{2} \mathrm{~d} y \\
& =\liminf _{r \rightarrow 0} \liminf _{n \rightarrow \infty} \int_{Q} \phi\left(\left[\frac{r}{\varepsilon_{n}}\right] y, M_{k_{n}}(y)\right)+\frac{1}{2}\left|\nabla \bar{U}_{n}(y)\right|^{2} \mathrm{~d} y \\
& \leq \liminf _{r \rightarrow 0} \liminf _{n \rightarrow \infty} \int_{Q} \phi\left(\left[\frac{r}{\varepsilon_{n}}\right] y, M_{k_{n}}(y)\right)+\frac{1}{2}\left|\nabla V_{k_{n}}(y)\right|^{2} \mathrm{~d} y \\
& =\liminf _{r \rightarrow 0} \liminf _{n \rightarrow \infty} \int_{Q} \phi\left(k_{n} y, M_{k_{n}}(y)\right)+\frac{1}{2}\left|\nabla u\left(x_{0}\right)+\nabla U_{k_{n}}(y)\right|^{2} \mathrm{~d} y \\
& \leq f_{\text {hom }}\left(m\left(x_{0}\right) ; \nabla u\left(x_{0}\right)\right)+\eta .
\end{aligned}
$$

Let $\eta$ goes to zero and we have the claim.

Step 4 (upper bound for arbitrary magnetizations). We now prove that for a general $m \in \mathcal{M}^{*}$ we have that

$$
\mathcal{F}^{-}(m ; D) \leq \int_{D} f_{\text {hom }}(m(x), \nabla u(x)) \text {. }
$$

Proof of Step 4. The Steps 2 and 3 ensure us that if $m \in \mathcal{M}^{*}$ is of the form (3.10) we have

$$
\mathcal{F}^{-}(m ; D)=\int_{D} f_{\text {hom }}(m(x), \nabla u(x))
$$

For a general $m \in \mathcal{M}^{*}$ construct a sequence $m_{k} \in \mathcal{M}^{*}$ of the form (3.10) such that $m_{k} \stackrel{*}{\rightarrow} m$ in $L^{\infty}\left(D ; \mathbb{R}^{3}\right)$ and $m_{k} \rightarrow m$ in $L^{2}\left(D, \mathbb{R}^{3}\right)$. We have, by (3.13) that

$$
\begin{aligned}
\mathcal{F}^{-}(m ; D) & \leq \liminf _{k \rightarrow \infty} \mathcal{F}^{-}\left(m_{k}, D\right) \\
& =\liminf _{k \rightarrow \infty} \int_{D} f_{\text {hom }}\left(m_{k}(x), \nabla u_{k}(x)\right),
\end{aligned}
$$

and up to extract a subsequence, we can assume that

$$
\liminf _{k \rightarrow \infty} \int_{D} f_{\text {hom }}\left(m_{k}(x), \nabla u_{k}(x)\right)=\lim _{k \rightarrow \infty} \int_{D} f_{\text {hom }}\left(m_{k}(x), \nabla u_{k}(x)\right) .
$$

Since by Lemma $2.2 \nabla u_{k} \rightarrow \nabla u$ in $L^{2}\left(D ; \mathbb{R}^{3}\right)$, using Fatou's Lemma and the upper semicontinuity of $f_{\text {hom }}$ (see Rem. 3.6) we obtain

$$
\begin{aligned}
\mathcal{F}^{-}(m ; D) & \leq \limsup _{k \rightarrow \infty} \int_{D} f_{\text {hom }}\left(m_{k}(x), \nabla u_{k}(x)\right) \\
& \leq \int_{D} \limsup _{k \rightarrow \infty} f_{\text {hom }}\left(m_{k}(x), \nabla u_{k}(x)\right) \\
& \leq \int_{D} f_{\text {hom }}(m(x), \nabla u(x))
\end{aligned}
$$

Last inequality together with Step 2 completes the proof. 
Now we can prove the main theorem

Proof of Theorem 1.1. We start proving that for any $\varepsilon_{n} \rightarrow 0^{+}$and $D \in \mathcal{O}(\Omega)$ we have

$$
\mathcal{F}^{-}(\cdot ; D)=\Gamma-\liminf _{n \rightarrow \infty} \mathcal{F}_{\varepsilon_{n}}(\cdot ; D) .
$$

Let $\left\{\varepsilon_{n_{k}}\right\}$ as in Lemma 3.3, we have always

$$
\mathcal{F}^{-}(\cdot ; D)=\Gamma-\liminf _{k \rightarrow \infty} \mathcal{F}_{\varepsilon_{n_{k}}}(\cdot ; D) \geq \Gamma-\liminf _{n \rightarrow \infty} \mathcal{F}_{\varepsilon_{n}}(\cdot ; D) .
$$

Thus assume by contradiction that there exists $\varepsilon_{n} \rightarrow 0^{+}$and $m \in \mathcal{M}^{*}$ such that

$$
\mathcal{F}^{-}(m ; D)>\Gamma-\liminf _{n \rightarrow \infty} \mathcal{F}_{\varepsilon_{n}}(m ; D),
$$

let $\left\{m_{n} \in \mathcal{M}\right\}$ be such that $m_{n} \stackrel{*}{\rightarrow} m$ in $L^{\infty}\left(D, \mathbb{R}^{3}\right)$ and $\mathcal{F}^{-}(m ; D)>\liminf \mathcal{F}_{\varepsilon_{n}}\left(m_{n}, D\right)$, and choose a subsequence $\left\{\varepsilon_{n_{j}}\right\}$ such that

$$
\mathcal{F}^{-}(m ; D)>\lim _{j \rightarrow \infty} \mathcal{F}_{\varepsilon_{n_{j}}}\left(m_{n_{j}}, D\right) .
$$

By previous lemmas we can extract a further subsequence $\left\{\varepsilon_{n_{j_{h}}}\right\}$ such that

$$
\begin{aligned}
\mathcal{F}^{-}(m ; D) & =\Gamma-\liminf _{h \rightarrow \infty} \mathcal{F}_{\varepsilon_{n_{j_{h}}}}(m ; D) \\
& \leq \lim _{h \rightarrow \infty} \mathcal{F}_{\varepsilon_{n_{j_{h}}}}\left(m_{n_{j_{h}}}, D\right) \\
& <\mathcal{F}^{-}(m ; D),
\end{aligned}
$$

which is a contradiction that prove (3.14).

Now we claim that for any $\varepsilon_{n} \rightarrow 0^{+}$and $m \in \mathcal{M}^{*}$

$$
\Gamma-\limsup _{n \rightarrow \infty} \mathcal{F}_{\varepsilon_{n}}(m ; D) \leq \mathcal{F}^{-}(m ; D) .
$$

By taking $m_{n}=m$ and using the boundedness of $\phi$, we get

$$
\begin{aligned}
\Gamma-\limsup _{n \rightarrow \infty} \mathcal{F}_{\varepsilon_{n}}(m ; D) & \leq \limsup _{n \rightarrow \infty} \mathcal{F}_{\varepsilon_{n}}\left(m_{n}, D\right) \\
& \leq C \int_{D}\left(|\nabla u|^{2}+1\right) d x=K,
\end{aligned}
$$

where $u \in H^{1}\left(\mathbb{R}^{3}\right)$ is the unique solution of $\Delta u+\operatorname{div} m=0$ in $\mathbb{R}^{3}$. Then in the definition of $\Gamma$ - lim sup we can consider only the sequences $\left\{m_{k}\right\}$ such that $m_{k} \stackrel{*}{\rightarrow} m$ in $L^{\infty}\left(\mathbb{R}^{3}\right)$ and

$$
\limsup _{n \rightarrow \infty} \mathcal{F}_{\varepsilon_{n}}\left(m_{n}, D\right) \leq K
$$

Moreover for every $\left\{m_{n}\right\}$ in this family we can always consider a subsequence $\left\{m_{n_{k}}\right\}$ with the same lim sup and with $\left\{\nabla u_{n_{k}}\right\}$ uniformly bounded in $l^{2}\left(\mathbb{R}^{3}\right)$ by a constant $l$.

Using the notation introduced in the proof of Lemma 3.3 we can conclude that

$$
\Gamma-\limsup _{n \rightarrow \infty} \mathcal{F}_{\varepsilon_{n}}(m ; D)=\Gamma\left(d_{l}\right)-\limsup _{n \rightarrow \infty} \mathcal{F}_{\varepsilon_{n}}(m ; D) .
$$


By Remark 3.4 and by Proposition 3.7, for any subsequence $\left\{\varepsilon_{n_{k}}\right\}$ of $\left\{\varepsilon_{n}\right\}$ there exists a subsequence $\left\{\varepsilon_{n_{k_{j}}}\right\}$ (depending on $D$ ) such that

$$
\begin{aligned}
\Gamma-\lim \mathcal{F}_{\varepsilon_{n_{k_{j}}}}(m ; D) & =\mathcal{F}^{-}(m ; D) \\
& =\int_{D} f_{\text {hom }}(m(x), \nabla u(x)) \mathrm{d} x .
\end{aligned}
$$

We can now apply the second part of Proposition 2.3 in the metric space $\left(B_{\infty} \times l B_{2}, d_{l}\right)$ to conclude that

$$
\Gamma\left(d_{l}\right)-\lim _{n \rightarrow \infty} \mathcal{F}_{\varepsilon_{n}}(m ; D)=\mathcal{F}^{-}(m ; D)
$$

Using (3.15) and (3.16) we have the claim.

Finally we have proved that $\mathcal{F}^{-}(m ; D)$ is in fact the $\Gamma-\lim _{\varepsilon \rightarrow 0} \mathcal{F}_{\varepsilon}(m ; D)$. Then applying this result with $D=\Omega$ and recalling that the non local term

$$
\frac{1}{2} \int_{\mathbb{R}^{3} \backslash \bar{\Omega}}|\nabla u(x)| \mathrm{d} x
$$

and the interaction energy

$$
\int_{\Omega} h_{e}(x) \cdot m(x) \mathrm{d} x
$$

are continuous perturbations, we have the desired result.

Acknowledgements. The author would like to thank Irene Fonseca for proposing him this argument of research, for the stimulating discussions and useful suggestions. As well he wants to thank Massimiliano Morini for helpful discussions and the hospitality of the Center for Nonlinear Analysis of CMU in Pittsburgh where part of this work was done. Finally he thanks the referee, whose comments helped him to improve the manuscript. This work was supported by PhD program in Mathematics of the University of Naples "Federico II" and partially by GNAMPA through the research project "Calcolo delle Variazioni, Teoria del Controllo e Ottimizzazione".

\section{REFERENCES}

[1] G. Anzellotti, S. Baldo and A. Visintin, Asymptotic behavior of the Landau-Lifshitz model of ferromagnetism. Appl. Math. Optim. 23 (1991) 171-192.

[2] J.M. Ball, A. Taheri and M. Winter, Local minimizers in micromagnetics and related problems. Calc. Var. Partial Differ. Equ. 14 (2002) 1-27.

[3] A. Braides and A. Defranceschi, Homogenization of multiple integrals. The Clarendon Press Oxford University Press, New York, Oxford Lecture Ser. Math. Appl. 12 (1998).

[4] A. Braides, I. Fonseca and G. Leoni, A-quasiconvexity: relaxation and homogenization. ESAIM: COCV 5 (2000) 539-577 (electronic).

[5] Jr. Brown and W. Fuller, Micromagnetics. Interscience Publishers, John Wiley \& Sons, New York, London (1963).

[6] B. Dacorogna, Direct methods in the calculus of variations. Appl. Math. Sci. 78 (1989).

[7] B. Dacorogna and I. Fonseca, A-B quasiconvexity and implicit partial differential equations. Calc. Var. Partial Differ. Equ. 14 (2002) 115-149.

[8] G. Dal Maso, An introduction to Г-convergence. Birkhäuser Boston Inc., Boston, MA Prog. Nonlinear Differ. Equ. Appl. 8 (1993).

[9] A. De Simone, Energy minimizers for large ferromagnetic bodies. Arch. Ration. Mech. Anal. 125 (1993) 99-143.

[10] A. De Simone, Hysteresis and imperfection sensitivity in small ferromagnetic particles. Meccanica 30 (1995) 591-603. Microstructure and phase transitions in solids (Udine, 1994).

[11] A. De simone, R.V. Kohn, S. Müller and F. Otto, A reduced theory for thin-film micromagnetics. Commun. Pure Appl. Math. 55 (2002) 1408-1460.

[12] A. De Simone, S. Müller, R.V. Kohn and F. Otto, A compactness result in the gradient theory of phase transitions. Proc. R. Soc. Edinb. Sect. A 131 (2001) 833-844. 
[13] I. Fonseca and G. Leoni, Relaxation results in micromagnetics. Ricerche Mat. 49 (2000) (suppl.) 269-304. Contributions in honor of the memory of Ennio De Giorgi (Italian).

[14] R.D. James and D. Kinderlehrer, Frustration in ferromagnetic materials. Contin. Mech. Thermodyn. 2 (1990) 215-239.

[15] L.D. Landau and E.M. Lifshits, Teoreticheskaya fizika. Tome VIII. "Nauka", Moscow, third edition (1992). Elektrodinamika sploshnykh sred. [Electrodynamics of continuous media], with a preface by Lifshits and L.P. Pitaevskiŭ, edited and with a preface by Pitaevskiı̌.

[16] L. Tartar, On mathematical tools for studying partial differential equations of continuum physics: $H$-measures and Young measures. Plenum, New York, in Developments in partial differential equations and applications to mathematical physics (Ferrara, 1991), (1992) 201-217.

[17] L. Tartar, Beyond Young measures. Meccanica 30 (1995) 505-526. Microstructure and phase transitions in solids (Udine, 1994).

[18] A. Visintin, On Landau-Lifshitz' equations for ferromagnetism. Japan J. Appl. Math. 2 (1985) 69-84. 